\title{
Properties, Preparations and Applications of Nano-sized Semiconductor Material
}

\author{
Chenlei Zhao ${ }^{1, \text { a }}$ \\ ${ }^{1}$ School of physics, Nanjing University, Nanjing 210093, China; \\ azhaochenlei502@sina.com
}

Keywords: Nano-sized, semiconductor, quantum effect, preparation

\begin{abstract}
The concept of Nano-sized semiconductor material and some of its basic properties such as small size effect, surface and interface effect, etc. are presented as well as how these basic properties explain its physics properties. This article also presents the preparative techniques and the applications of Nano-sized semiconductor material.
\end{abstract}

\section{Introduction}

In the normal semiconductor material, the size of the material is much greater than an electron's de Broglie wavelength while in the Nano-sized semiconductor, when the length of one of its three dimensions is near an electron's de Broglie wavelength, the movement of an electron will be quantized so its energy will be restricted to several separated eigenstates. In this case, the way we used to study the electron in normal materials is no longer appropriate. The characters of Nano-sized semiconductor's structure (the size of its atomic domain is less than $100 \mathrm{~nm}$ etc.) are the main causes of its special physics properties. These physics properties make the Nano-sized semiconductor materials ideal materials in many areas. For example, Nano-sized $\mathrm{TiO}_{2}, \mathrm{ZnO}, \mathrm{Fe}_{2} \mathrm{O}_{3}, \mathrm{WO}_{3}$, etc. have been used in the Nanocrystalline photovoltaic cell. And Nano-sized $\mathrm{BaTiO}_{3}$ has been used in many kinds of sensors. Also the research in the the preparative techniques of Nano-sized semiconductor material is very hot nowadays. This article discusses some physics properties of Nano-sized semiconductor material as well as its preparative techniques and applications[1].

\section{Basic Properties of Nanoparticles.}

Small Size Effect: When the size of a particle is near or smaller than an electron's de Broglie wavelength or light wave's wavelength, its periodic boundary conditions will be breached. As a result, some of its acoustic, optical, electromagnetic, thermal properties will show some brand new small size effects. Some applications have been designed based on this effect. For example, some microwave absorbing materials can be made based on the property that the resonance frequency shift of plasma will change according to the size of the particle. And this property has been used on the manufactural of stealth aircraft and electromagnetic wave shielding[2].

Surface and Interface Effect: The phenomenon that the proportion of the surface atoms' number of the nanoparticle to the total atoms' number will increase rapidly with the decrease of the nanoparticle's size is called surface and interface effect. This also causes the increase of the surface energy and the surface tension. As a result, some of its optical, electromagnetic and chemical properties will change[1]. Also, the crystalline field environment and the binding energy of the surface atoms are different from those of the inner atoms. Thus the chemical activity of the surface atoms is much higher, which affects the properties of the material.

Quantum Size Effect: The quantum size effect is a phenomenon that when the size of a particle is reduced to a particular number, the electronic energy level around the Fermi level will become separated. And there will be discontinuous highest occupied molecular orbital and lowest unoccupied molecular orbital. When the change in the energy level is greater than the change of thermal energy, optical energy, or electromagnetic energy, there will be some significant differences from ordinary materials in acoustic, optical, electromagnetic, and thermal properties of nanoparticles. 
Macroscopic quantum tunneling effect: The ability of microscopic particles to penetrate potential barrier even when their energy is lower than the height of the potential barrier is called tunneling effect. In recent years, scientists have found that some macroscopic quantities such as the magnetization of microscopic particles also have tunneling effect, and this is called macroscopic quantum tunneling effect. The macroscopic quantum tunneling effect will be the foundation of the microelectronic device and the optoelectronic device in the future because it sets a limit to the existing microelectronic devices' degree of miniaturization. When the microelectronic devices want to get more miniaturization, this effect must be considered.

\section{Physics Properties of Nano-sized Semiconductor Material}

Optical Property: As a result of the quantum size effect we discussed above, Nano-sized semiconductor particle's optical physical and optical chemical properties are one of the most active research areas. As we discussed above, when the size of a Nano-sized semiconductor particle is smaller than the exciton Bohr radius, with the decrease of the size of the Nano-sized semiconductor particle, its energy efficiency gap will increase. Clearly, as a result, there will be a blue shift in the absorption spectrum and the fluorescence spectrum. The quantum size effect of some Nano-sized semiconductor particles can be expressed clearly by the Brus Formula[3]:

$$
\mathrm{E}(\mathrm{r})=E_{g}+\frac{h^{2} \pi^{2}}{2 \mu r^{2}}-1.786 \frac{e^{2}}{\varepsilon r}-0.248 E_{R y}
$$

In this formula, $E(r)$ means the energy absorption gap of Nano-sized particle, $r$ represents the radios of the particle, $\mu$ is the effective quality and $E g$ is the energy absorption gap of normal materials. From this formula, we can tell that with the decrease of the particle's radius, the energy efficiency gap will increase, which will cause a blue shift in the absorption spectrum[4]. Also, some researchs have found out that when covered with surfactant molecular, $\mathrm{SnO}_{2}$ nanoparticle will have a red shift in the absorption spectrum caused by dielectric confinement effect, and with the increase in the difference between the dielectric constant of the surface coverings and $\mathrm{SnO}_{2}$, the quantity of red shift will increase[5].

Electrical Properties: Dielectric property and piezoelectric property are two of the most important material's properties. The dielectric property and piezoelectric property of Nano-sized semiconductor material are very different from those of normal semiconductor material. It can be basically summarized as follows:

The dielectric constant of Nano-sized semiconductor material will increase with the decrease of measuring frequency, while that of the normal semiconductor material is usually smaller. The tendency of increase in the low frequency area is far less significant.

In the low frequency area, the dielectric constant of Nano-sized semiconductor material shows some small size effect. When the diameter of the particle is very small, the dielectric constant is also small, and with the diameter increasing, the dielectric constant will increase and then decrease again, so there will be a maximum value at a critical dimension.

For some Nano-sized semiconductor materials, there are many dangling bonds on the interface, causing a change in the distribution of charge, so that there will be some dipole moment. If the outside pressure makes a change in the direction of the dipole moment, there will be macroscopical charge accumulation, so that there will be some significant piezoelectric effects[2].

\section{Preparative Technique of Nano-sized Semiconductor Material}

Sol-gel Method: Sol-gel method is to produce Nano-sized material by mixing the active chemical presoma and then hydrolyze them into sol, polymerize into gel, then dry and frit them into Nano-sized material. It can make the material uniform on the molecular level. It has the advantages of low reaction temperature, low cost and it is easy to be handled. But this method also has some 
disadvantages. Some of the raw materials are organic and toxic. Also this reaction will need a long time to finish, from a couple of days to a couple of weeks[1].

Microemulsion Method: Use surface active agent to mix two kinds of mutually incompatible solvent into emulsion and then go through a nucleation process, an agglomeration process and a heat treatment to make them into Nano-sized particles[6]. This method has the following advantages: (1) The distribution of the particle's diameter is very narrow so that it can be controlled precisely. (2) Using different kinds of surface active agents to modify the surface of the particle can get some Nano-sized particles with specific properties. (3) There is one or a couple of layers of surface active agent on the surface of the particle so that the particles won't coalesce.

Molecular Beam Epitaxy (MBE): MBE is a new technique to grow high quality crystal film on crystal substrate. In a highly vacuumed condition, heat the furnace with all raw materials to generate steam. And then make them go through a little hole to become a collimation of atomic beam or molecular beam. Spray the beam directly to a single crystal substrate of an appropriate temperature and control the scan of the beam at the same time. At last, the atoms or molecules will grow on the substrate layer by layer to become a film[7]. The rate of growing is very slow so that the thickness of the film can be controlled very precisely. Also, MBE is a kinetic process rather than a thermodynamic process so it can grow some films that the normal thermal equilibrium way cannot $[8,9,10]$.

Metal Organic Chemical Vapor Deposition (MOCVD): MOCVD is another technique developed at the same time as MBE. It uses hydrogen to transport metalorganic vapor and nonmetal hydride to the surface of heated substrate in the reaction chamber and go through thermal decomposition to grow epitaxial layer eventually[7]. The deposition temperature is very low and it has a wide application. It can almost grow all kinds of compounds and alloy semiconductors. So it's suitable for mass production. But some of the metalorganic vapor are toxic and easy to burn. Also, because the reaction temperature is very low, some reactions may take place in the gas phase[11,12].

\section{Applications of Nano-sized Semiconductor Material}

Nanocrystalline photovoltaic cell: It has been proved both theoretically and experimentally that the photovoltaic cell based on the normal semiconductor material is not able to transcend the transfer limit. However, Nano-sized semiconductor material has many different optical and electromagnetic properties compared with normal materials. It can be used to produce photovoltaic cells with high efficiency especially the materials with dye sensitization. Also the preparation of the nanocrystalline photovoltaic cell is simple and has a high efficiency of the surface charges' transfer. Since Gratzel first reported the high efficiency of nanocrystalline photovoltaic cell with dye sensitization, many scientists have focused on this area and the research of this area is becoming hotter[2].

Sensors: As a result of the large specific surface area, high activity and many specific properties, Nano-sized semiconductor material is an ideal material for many kinds of sensors. With the change of the light, temperature or humidity, the transport of valence electrons will also change and macroscopically it will act as a change in the electric resistance. The advantages of the sensors made from Nano-sized semiconductor material are that they will have a high sensitivity and a short reacting time.

\section{Summary}

The 21st century is an information age, and many prototypes of information devices lie in the Nano-sized semiconductor material. Although the research in Nano-sized semiconductor material is just starting, I believe its specific properties will make it one of the most useful materials in the future. There sure will be new breakthroughs in this area. 


\section{References}

[1]Luan Yemei, An Maozhong. Properties and Preparation of Semiconductor Nanomaterials [J]. Semiconductor Optoelectronics, 2008 (6).

[2] Li Xinyong, Li Shuben. Progress in Nanometer Semiconductor Materials [J]. Progress in Chemistry, 1996, 8(3): 231-239.

[3] Brus L E. Electron-electron and electron - hole interactions in small semiconductor crystallites: The size dependence of the lowest excited electronic state[J]. The Journal of chemical physics, 1984, 80(9): 4403-4409.

[4]Guan Baiou, Han Guanyun. The Optical Properties and Progress of Nanosize Semiconductor Materials [J]. Journal of Optoelectronics • Laser, 1998, 9(3): 260-263.

[5]Yu Baolong, Wu Xiaochun, Chen Wenju, et al. Optical Properties of $\mathrm{SnO}_{2}$ Nanometer Particles [J]. Acta Physica Sinica, 1995, 44(4): 660-665.

[6] Motte L, Billoudet F, Lacaze E, et al. Self-organization into 2D and 3D superlattices of nanosized particles differing by their size[J]. The Journal of Physical Chemistry B, 1997, 101(2): 138-144.

[7]Wang Zhanguo. The Fabrication and Characterization on Nano-scale Semiconductor Materials [J]. Micronanoelectronic Technology, 2002, 39(1): 9-14.

[8] Arthur Jr J R. Interaction of Ga and As2 molecular beams with GaAs surfaces[J]. Journal of Applied Physics, 1968, 39(8): 4032-4034.

[9] Cho A Y, Arthur J R. Molecular beam epitaxy[J]. Progress in solid state chemistry, 1975, 10: 157-191.

[10] Razeghi M, Tsang W T. Semiconductors and Semimetals, Vol[J]. 1985.

[11] Kerr T. Picogiga multiwafer MBE system[J]. III-Vs Review, 1991, 4(3): 20-22.

[12] Manasevit H M. Single-Crystal Gallium Arsenide on Insulating Substrates[J]. Applied Physics Letters, 1968, 12(4): 156-159. 